\title{
Thermodynamics and structure of simple liquids in the hyperbolic plane
}

\author{
François Sausset, Gilles Tarjus and Pascal Viot \\ Laboratoire de Physique Théorique de la Matière Condensée, Université Pierre et \\ Marie Curie-Paris 6, UMR CNRS 7600, 4 place Jussieu, 75252 Paris Cedex 05, France \\ E-mail: \\ sausset@lptmc.jussieu.fr, tarjus@lptmc. jussieu.fr, viot@lptmc. jussieu.fr
}

\begin{abstract}
We provide a consistent statistical-mechanical treatment for describing the thermodynamics and the structure of fluids embedded in the hyperbolic plane. In particular, we derive a generalization of the virial equation relating the bulk thermodynamic pressure to the pair correlation function and we develop the appropriate setting for extending the integral-equation approach of liquid-state theory in order to describe the fluid structure. We apply the formalism and study the influence of negative space curvature on two types of systems that have been recently considered: Coulombic systems, such as the one- and two-component plasma models, and fluids interacting through short-range pair potentials, such as the hard-disk and the LennardJones models.
\end{abstract}




\section{Introduction}

When a system is embedded in a space of nonzero curvature, its long-distance properties, such as the thermodynamic quantities and the critical behavior, are strongly modified. A uniform positive curvature, characteristic of spherical geometry, has the drawback of leading to spaces of finite extent. Genuine long-distance properties on the other hand are accessible in hyperbolic spaces characterized by a constant negative curvature. The influence of the negative curvature of hyperbolic space on critical phenomena has been investigated both for hard spins on hyperbolic lattices [1, 2, 3, 4, 15, 6, 17, 8, 9, 10, 11, 12, 13] and in a continuum field-theoretical description [14, 15, 15, 16].

In brief, the presence of an intrinsic characteristic length associated with the metric makes the critical behavior of Ising-type systems mean-field-like[1, 2, 4, 15, 6, 8] and drastically alters, if not suppresses, critical points in models with a continuous symmetry [14, 16, 9, 11] In the last decade, the thermodynamics of simple fluids in the hyperbolic plane has also received special attention. This is the case for systems interacting through the Coulomb potential, such as the one- and two-component plasma models [17, 18, 19] and for the hard-disk fluid[20, 21, 22].

In addition to being a mathematical curiosity and providing simple systems for studying the influence of space curvature on long-distance properties, fluids in the hyperbolic plane have been considered in the context of jamming phenomena and glass formation in liquids. The rationale behind this comes from the concept of "geometric frustration" that describes an incompatibility between the preferred local order and the tiling of the whole space [23, 24]. Geometric frustration has emerged in the theoretical description of glasses and amorphous solids from the consideration of local icosahedral order in metallic glasses. No such frustration however operates in assemblies of monodisperse spherical particles in ordinary ("flat") two-dimensional space, and Nelson and coworkers [25, 26, 24] have proposed to curve two-dimensional space in order to introduce frustration and thereby mimic glasses in three-dimensional space. Two recent investigations have been carried out along these lines. Modes and Kamien[20, 21] have focused on isostatic packings in hyperbolic space, the isostatic property having been argued to play an important role in the physics of nearly jammed random packings of particles in Euclidean three-dimensional space 27]. On the other hand, building on a frustration-based approach of the glass transition[28, 29], we have studied by molecular dynamics simulation the slowing down of the dynamics with decreasing temperature in a monatomic liquid: a truncated Lennard-Jones model in the hyperbolic plane 30 .

In the present work, we provide a consistent statistical-mechanical treatment for describing the thermodynamics and the structure of fluids embedded in the hyperbolic plane. The paper is organized as follows. In section 2, we introduce the statisticalmechanical framework for systems in the hyperbolic plane. By using a variant of the Green-Bogoliubov method, we relate the bulk thermodynamic pressure to the pair distribution function. In section 3, we extend the integral-equation approach, which 
is commonly used in liquid-state theory, to calculate the pair correlation function in hyperbolic geometry. We discuss thermodynamic consistency and gas-liquid critical behavior. In section 4, we focus on Coulombic systems, such as the one- and twocomponent plasma models. We apply the formalism developed in the previous sections to two cases for which the pair correlation functions are known exactly: the limit of high temperature and a special value of the temperature. In section 5, we present the numerical solution of standard approximate integral equations of liquid-state physics (namely, the Percus-Yevick and hypernetted chain equations) that have been obtained after making use of the harmonic analysis in the hyperbolic plane. We consider the hard-disk fluid and the (truncated) Lennard-Jones liquid and compare with existing simulation data. Finally, section 6 contains our concluding remarks, and some technical details are provided in two appendices.

\section{The generalized virial equation of state}

For an atomic liquid at equilibrium in the grand-canonical ensemble (fixed temperature $T$, total area $A$ and chemical potential $\mu$ ), the partition function is given by

$$
\Xi(T, A, \mu)=\sum_{N=1}^{\infty} \frac{\left(Z_{1} e^{\beta \mu}\right)^{N}}{N ! A^{N}} \int \mathrm{d} \mathbf{A}^{N} \exp \left(-\beta \sum_{i=1}^{N} \sum_{j>i} u\left(r_{i j}\right)\right)
$$

where $u(r)$ is the pair potential with $r$ the appropriate interatomic geodesic distance (for simplicity, we only consider pairwise additive interactions), $\beta=1 /\left(k_{B} T\right)$, and $Z_{1}(T, A)$ is the partition function of a single particle, i.e. the ideal gas contribution. The above expression is valid for any two-dimensional space with $d A$ the appropriate infinitesimal surface area. It therefore applies as well to the hyperbolic plane, with however some subtelties that are discussed below.

The hyperbolic plane $H^{2}$ is a two-dimensional manifold of constant negative curvature, whose metric in polar coodinates $(r, \phi)$ is given by

$$
\mathrm{d} s^{2}=\mathrm{d} r^{2}+\left(\frac{\sinh (\kappa r)}{\kappa}\right)^{2} \mathrm{~d} \phi^{2} .
$$

The Gaussian curvature of $H^{2}$ is $-\kappa^{2}$. The curvature parameter $\kappa>0$ therefore measures the deviation from "flat" (Euclidean) space; by an abuse of language, we shall sometimes refer to $\kappa^{-1}$ as the "radius of curvature". (Note that $H^{2}$ is not embeddable in three-dimensional Euclidean space and is usually described through representations such as the Poincaré disk model that is a conformal projection of $H^{2}$ onto a disk of radius $\kappa^{-1}$.) In polar coordinates, the differential area is expressed as

$$
d A=\frac{\sinh (\kappa r)}{\kappa} d r d \phi
$$

The hyperbolic plane is of infinite extent but the exponential character of the metric at large distance (see Eqs. (2) and (3) ) induces the peculiar property that the boundary of a finite region of $H^{2}$ grows as fast as the total area of this region when the latter 
increases. A simple illustration is provided by a disk of radius $r$ : from Eq. (3), one finds that its area is equal to $A(r)=2 \pi(\cosh (\kappa r)-1) / \kappa^{2}$ whereas its perimeter is given by $P(r)=2 \pi \sinh (\kappa r) / \kappa$, so that for large $r$, both $A(r)$ and $P(r)$ grow as $\exp (\kappa r)$.

As a consequence of the above property, boundary effects are never negligible, even in the thermodynamic limit. Generally speaking, the statistical mechanics of fluids on $H^{2}$ involves multiple integral over space, as in Eq. (1). Integration is performed on both the "bulk" and the "boundary region" of the system, the latter being taken as a region of finite width (or a width whose ratio to the linear size of the system goes to zero when the latter goes to infinity) near the boundary in an otherwise very large sample. The boundary contribution of course depends on the boundary condition imposed on the system, but it is never negligible compared to the bulk contribution. This is already true for the ideal-gas limit. The canonical partition function $Z_{1}$ is proportional to the total area $A$; however, the proportionality constant is not a pure bulk property and depends on the type of boundary condition[21.

In this work, we are only interested in the bulk behavior of fluids in $H^{2}$, which is what can be directly compared to fluids in Euclidean space. One way to eliminate unwanted boundary effects in $H^{2}$ is to consider periodic boundary conditions [31]. This has been done for instance by Modes and Kamien for calculating the properties of a gas of hard disks in the low-density limit near ideal behavior[21]. We shall take here a simpler route that has already been followed in previous studies of spin and field-theoretical models in hyperbolic geometry[1, 4, 15]: we implicitly restrict all integrals appearing in any statistical-mechanical expression to the "bulk" of the sample, i.e. the fluid far enough from the boundary. All resulting thermodynamic and structural quantities will therefore be dubbed "bulk" ones. We stress again that the present problem associated with the thermodynamic limit in hyperpolic geometry is a general one which is not related to the presence of long-range correlations between particles. (This point will be addressed further down.)

From the (bulk) grand-canonical partition function in Eq. (1), one obtains the (bulk) thermodynamic pressure as

$$
\beta P=\left.\frac{\partial \ln \Xi}{\partial A}\right|_{T, \mu}
$$

For short-range interactions, the low-density expansion of the equation of state can be put in the usual form[21,

$$
\frac{\beta P}{\rho}=1+\sum_{j \geq 2} B_{j}(T) \rho^{j-1},
$$

where $\rho=N / A$ and $B_{j}$ is the $j$ th virial coefficient. When restricting the calculation to the bulk of the system, the second virial coefficient $B_{2}$ can be expressed as

$$
B_{2}=-\frac{1}{2} \int d A f(r)
$$

where $d A$ is given by Eq. (3) and $f(r)=\left(e^{-\beta u(r)}-1\right)$ is the Mayer function. (In the calculation of $B_{2}$ for short-range interactions, it is easy to see that one obtains the 
same result by restricting the system to the bulk region and by using periodic boundary conditions[21].) As a result,

$$
B_{2}=\pi \int_{0}^{\infty} d r \frac{\sinh (\kappa r)}{\kappa} f(r),
$$

which for hard disks of diameter $\sigma$ simplifies to 21$]$

$$
B_{2}=\pi \frac{(\cosh (\kappa \sigma)-1)}{\kappa^{2}} .
$$

The above expression correctly reduces to the Euclidean result $B_{2}=\pi \sigma^{2} / 2$ when $\kappa \rightarrow 0$.

To go beyond the low-density regime and describe the liquid phase, a natural strategy is to relate the pressure to the pair distribution function 32 . However, the hyperbolic geometry prevents a direct derivation through the Clausius function [32]. We have instead used a variant of the Green-Bogoliubov method[33]. To calculate the derivative in Eq. (4), we choose to perform an affine transformation of the elementary area element,

$$
d A^{\prime}=(1+\xi) d A
$$

with $\xi$ an infinitesimal parameter. Inserting Eq. (3) in Eq. (9) yields the following transformation of the radial coordinate $r$ to first order in $\xi$ :

$$
r^{\prime}=r+\xi \frac{(\cosh (\kappa r)-1)}{\kappa \sinh (\kappa r)} .
$$

The presence of curvature leads to a nonlinear transformation of the coordinate, contrary to what occurs in flat Euclidean space[34]. We then consider the infinitesimal variation of $\ln (\Xi)$ generated by the affine transformation,

$$
\delta \ln (\Xi)=\xi\langle N\rangle-\frac{\beta}{2} \int d A_{1} \int d A_{2} \rho^{(2)}\left(\mathbf{r}_{1}, \mathbf{r}_{2}\right) \delta u\left(r_{12}\right),
$$

where $\rho^{(2)}\left(\mathbf{r}_{1}, \mathbf{r}_{2}\right)$ is the (bulk) two-particle density. By using Eq. (10), the infinitesimal increase of the potential is found equal to

$$
\delta u(r)=\xi \frac{\cosh (\kappa r)-1}{\kappa \sinh (\kappa r)} u^{\prime}(r)
$$

where $u^{\prime}(r)$ denotes the derivative of $u(r)$ with respect to $r$.

Taking advantage of the homogeneity and the isotropy of $H^{2}$ (far from the boundary), we then obtain the bulk thermodynamic pressure as

$$
\frac{\beta P}{\rho}=1-\frac{\pi \beta \rho}{\kappa^{2}} \int_{0}^{\infty} d r g(r)(\cosh (\kappa r)-1) u^{\prime}(r)
$$

where we have used that $\rho^{(2)}\left(\mathbf{r}_{1}, \mathbf{r}_{2}\right)=\rho^{2} g(r), g(r)$ being the bulk radial distribution function[32]. This result generalizes the virial equation of state obtained in Euclidean space. The latter is recovered when taking the $\kappa \rightarrow 0$ limit in Eq. (13):

$$
\frac{\beta P}{\rho}=1-\frac{\pi \beta \rho}{2} \int_{0}^{\infty} d r r^{2} g(r) u^{\prime}(r) .
$$

At large distance, $g(r)$ goes to 1 , and Eq. (13) shows that the pressure is only defined if $\int_{R}^{\infty} d r(\cosh (\kappa r)-1) u^{\prime}(r)$ is finite, with $R$ some irrelevant cut-off distance. This 
imposes that the pair potential $u(r)$ decays faster than $\exp (-\kappa r)$ when $r \rightarrow \infty$. This requirement prevents using any interaction potential with an algebraic decay, contrary to the $d$-dimensional Euclidean case where all potentials $u(r) \propto r^{-n}$ with $n \geq d$ lead to a well-defined thermodynamic limit (for the Coulombic interaction, see below). The above condition is of course satisfied for hard disks, but also for the one- and twocomponent plasma models because, as will be discussed later, the Coulomb potential decreases exponentially fast at large distance in the hyperbolic plane.

In the case of hard disks, after introducing the auxiliary function $y(r)=g(r) e^{\beta u(r)}$, we can express the pressure as

$$
\frac{\beta P}{\rho}=1+\frac{\pi \rho}{\kappa^{2}} \int_{0}^{\infty} d r(\cosh (\kappa \sigma)-1) y(r) \frac{d}{d r} \exp (-\beta u(r))
$$

and using $\frac{d}{d r} \exp (-\beta u(r))=\delta(r-\sigma)$ finally leads to

$$
\frac{\beta P}{\rho}=1+\frac{\pi \rho}{\kappa^{2}}(\cosh (\kappa \sigma)-1) g\left(\sigma^{+}\right)
$$

At low density, the radial distribution function at contact $g\left(\sigma^{+}\right)$goes to one, so that one recovers the virial expansion in Eq. (5) to first order with the second virial coefficient given by Eq. (8) .

\section{Integral equations}

In liquid-state theory, approximate integral equations are a standard tool to describe the structure and the thermodynamics of a system. The approach is based on the (exact) Ornstein-Zernike equation that relates the radial distribution function $g(r)$, or more precisely the so-called pair correlation function $h(r)=g(r)-1$, to the direct correlation function $c(r)$ which is obtained from the second functional derivative of the grand potential with respect to local density fluctuations [32, 35]. In the hyperbolic plane, the Ornstein-Zernike equation reads

$$
h(r)=c(r)+\rho \int d A^{\prime} h\left(r^{\prime}\right) c\left(t\left(\mathbf{r}, \mathbf{r}^{\prime}\right)\right)
$$

where $t\left(\mathbf{r}, \mathbf{r}^{\prime}\right)$ is the modulus of the displacement associated with an element of the hyperbolic translation group 36]. (In Euclidean space, $t\left(\mathbf{r}, \mathbf{r}^{\prime}\right)=\left|\mathbf{r}-\mathbf{r}^{\prime}\right|$.) Again, Eq. (17) should be considered as a bulk result, in which boundary effects have been removed when taking the thermodynamic limit.

The Ornstein-Zernike equation is more conveniently expressed after applying a Fourier-Helgason transform (that generalizes the Fourier transform of the Euclidean space):

$$
\tilde{h}(k)=\tilde{c}(k)+\rho \tilde{c}(k) \tilde{h}(k) .
$$

Note that for an isotropic function $h(r)$ as considered here, the Fourier-Helgason transform becomes a Mehler-Fock transform[37]. For more details, see Appendix A.

The basis of the integral-equation approach for liquids is that the direct correlation function $c(r)$ has a simpler structure and is shorter-ranged than $h(r)$ and, as a 
consequence, is a better starting point for approximations. Common approximations are the Percus-Yevick (PY) and hypernetted chain (HNC) closures in which the direct correlation function is taken as 32

$$
\begin{aligned}
& c(r)=(1+\gamma(r))(\exp (-\beta u(r))-1) \quad(\mathrm{PY}) \\
& c(r)=\exp (-\beta u(r)+\gamma(r))-(1+\gamma(r))(\mathrm{HNC}),
\end{aligned}
$$

where $\gamma(r)=h(r)-c(r)$.

In Euclidean geometry and for hard spheres, the PY closure (the direct correlation function is then zero beyond $\sigma$ ) is amenable to an analytical solution in spaces of odd dimensions [32, 38, 39, 40, 41, 42] and compares rather well with computer simulation results. Recently, a semi-analytical solution of the PY integral equation has also been obtained in two-dimensional Euclidean space [43, 44]: the first ten virial coefficients have been calculated and compared to Monte Carlo results. In addition, the PY approximation for hard discs has been numerically solved on a sphere [45].

Due to the approximations involved in the integral-equation approach, going from the structure of the fluid to its (bulk) thermodynamic properties depends on the route chosen. Once the pair correlation function is known, one may find the pressure with the help of the virial equation, Eq. (13), but also through the compressibility relation or that for the excess internal energy[32]. For instance, the compressibility relation reads

$$
\rho k_{B} T \chi_{T}=1+\rho \int d A h(r)
$$

where again only bulk contributions are considered.

At this point, it is important to stress a peculiar feature of the Fourier-Helgason transform: contrary to what occurs in Euclidean space, $\int d A h(r) \neq \tilde{h}(0)$. Indeed, by using the results of Appendix A, one finds that

$$
\begin{aligned}
\tilde{h}(0) & =\frac{2 \pi}{\kappa} \int_{0}^{\infty} d r \sinh (\kappa r) P_{-1 / 2}(\cosh (\kappa r)) h(r) \\
& \neq \frac{2 \pi}{\kappa} \int_{0}^{\infty} d r \sinh (\kappa r) h(r),
\end{aligned}
$$

where $P_{-1 / 2}(x)$ is a Legendre function of the first kind (conical function). As a consequence, the bulk compressibility in $H^{2}$ is not given by the $k=0$ value of the Fourier-Helgason transform of the pair correlation function. On the other hand, neglecting the boundary effects, one still has

$$
\rho k_{B} T \chi_{T}=\frac{1}{1-\rho \int d A c(r)},
$$

so that the bulk pressure can be obtained by a thermodynamic integration according to

$$
\frac{\beta P}{\rho}=1-\frac{1}{\rho} \int_{0}^{\rho} d \rho^{\prime} \rho^{\prime} \int d A c\left(r ; \rho^{\prime}\right)
$$

where $c(r, \rho)$ denotes the direct correlation function at the density $\rho$. 
For the hard-sphere fluid in Euclidean space, the Percus-Yevick integral equation provides a very accurate equation of state when the pressure is calculated with the following empirical rule 32$]$ :

$$
P=\frac{2 P_{c}+P_{v}}{3}
$$

where $P_{c}$ and $P_{v}$ denote the "compressibility" and "virial" pressures obtained from Eqs. (24) and (14), respectively.

Note finally that according to Eq. (21), a finite compressibility implies that $\int d A h(r)$ is finite, which in turn requires that $h(r)$ decays at large distance faster than $\exp (-\kappa r)$. A gas-liquid critical point with a diverging compressibility may thus occur with a pair correlation function that decreases exponentially as $\exp (-\kappa r)$. Assume for instance that $h(r)$ behaves as $\exp (-r / \xi)$ at large distance. One then finds, with again the restriction that the integration is only over the bulk region far from the boundary, that

$$
\int_{r<L} d A h(r) \sim \frac{(\kappa \xi)^{2}}{1-(\kappa \xi)^{2}}\left[1-\left(\frac{1+\kappa \xi}{2 \kappa \xi}\right) e^{-\left(\frac{1}{\xi}-\kappa\right) L}\right]+r e g,
$$

when $L \rightarrow+\infty$, where reg denotes regular, finite terms. The above expression diverges when $\kappa \xi \rightarrow 1^{-}$. If $\xi$ is a regular function of $T$ and $\rho$ when $\xi<\kappa^{-1}$, this suggests that the critical behavior found near to $\xi_{c}=\xi\left(T_{c}, \rho_{c}\right)=\kappa^{-1}$ is of mean-field type, with classical exponents. In particular, the bulk compressibility $\chi_{T}$ would diverge as $1 /\left(1-(\kappa \xi)^{2}\right) \sim\left(T-T_{c}\right)^{-1}$, implying that the critical exponent $\gamma=1$. In addition, assuming that $h(r) \sim \exp (-\kappa r)$ at large distance at the critical point and using the known expression of the Fourier-Helgason transform of $1 / \cosh (\kappa r)$ [37], it is easy to derive that the Fourier-Helgason transform $\tilde{h}(k)$ of the pair correlation function is finite at $k=0$, even if the compressibility diverges (which is a dramatic illustration of Eq. $(22))$, and has a regular expansion in $(k / \kappa)^{2}$ when $(k / \kappa) \rightarrow 0$.

The expected mean-field nature of the gas-liquid critical point in $H^{2}$ has the very same origin as the mean-field character of the critical point of the Ising model in hyperbolic geometry[1, 4, 15]: the divergence of the compressibility/susceptibility

is controlled by the exponential growth with distance of the differential surface area associated with the hyperbolic metric. An approximate description of the gas-liquid critical behavior will also be provided in section 5.2 .

\section{Coulombic systems}

We now consider systems of particles interacting through a Coulomb potential, as was done before by Jancovici and coworkers[19, 18, 17]. For a monodisperse system (particles with equal charges of the same sign), it is necessary to add a charged uniform background such that the global electroneutrality is satisfied, thereby allowing a proper thermodynamic limit. In the limit of point-like particles, this corresponds to the onecomponent plasma model (OCP). When considering a binary mixture of two species of oppositely charged particles (with charges $\pm q$ ) in equal concentrations, it is possible to 
obtain a well-defined thermodynamic limit without introducing a charged background: in the limit of point-particles, this is the two-component plasma model (TCP). In this latter case, the system is stable at high temperature, down to a temperature at which a collapse of pairs of opposite charges occurs. When the charged particles have an additional hard-core interaction and at low charge density, the system undergoes a Kosterlitz-Thouless transition at a lower temperature $T_{c}=q^{2} /\left(4 k_{B}\right)$ [46, 47].

The Coulomb potential is formally defined as the solution of the Poisson equation, which is related to the Green's function of the following inhomogeneous Laplace equation:

$$
\Delta v(r, \phi)=-2 \pi \delta^{(2)}(r, \phi),
$$

where $\Delta$ is the Laplacian operator and $\delta^{(2)}(r, \phi)$ the Dirac distribution in the appropriate manifold. In the Euclidean plane and for a function depending on the radial coordinate only, $\Delta$ is given by

$$
\Delta v(r)=\frac{1}{r} \frac{\partial}{\partial r}\left(r \frac{\partial v(r)}{\partial r}\right)
$$

and the solution of Eq. (27) is

$$
v(r)=-\ln (r / L)
$$

where $L$ is an arbitrary length. Therefore in two dimensions, the Coulombic interaction potential diverges with the distance, even if the associated force remains a (slow) decreasing function of the distance.

On the other hand, in the hyperbolic plane, the Laplacian acts on a function of the radial coordinate as

$$
\Delta v(r)=\frac{1}{\sinh (\kappa r)} \frac{\partial}{\partial r}\left(\sinh (\kappa r) \frac{\partial v(r)}{\partial r}\right) .
$$

The solution of the inhomogeneous Laplace equation is then given by

$$
v(r)=-\ln \left(\tanh \left(\frac{\kappa r}{2}\right)\right),
$$

so that the pair interaction between two point particles with charges $q_{1}$ and $q_{2}$ at a geodesic distance $r$ is equal to $q_{1} q_{2} v(r)$ with $v(r)$ given above. The curvature of the hyperbolic plane thus introduces a screening of the Coulomb interaction. Indeed, $v(r)$ now decreases as $\exp (-\kappa r)$ at large distance beyond $\kappa^{-1}$.

It is possible to derive analytical expressions for the thermodynamic quantities in two cases: the high-temperature limit, where the Debye-Hückel approximation is asymptotically exact, and the particular value of the temperature $T=q^{2} /\left(2 k_{B}\right)$, where a large amount of exact results have been obtained both in the Euclidean and in the hyperbolic plane. In the following we shall consider the two models introduced above, the OCP and the TCP. (To extend the investigation beyond the two cases considered here, one could use the HNC integral equation which is known to give a good description of the pair correlation function in flat space 32, but this is left for future work.) 


\subsection{One-component plasma (OCP)}

For the OCP, the charged background provides a uniform neutralizing contribution. Assuming a perfect compensation amounts to replace $g(r)$ by $h(r)=g(r)-1$ in the expression of the virial equation of state. After taking the derivative of Eq. (31), the equation of state can be written as

$$
\frac{\beta P}{\rho}=1+\frac{\pi \beta q^{2} \rho}{\kappa} \int_{0}^{\infty} d r h(r) \frac{(\cosh (\kappa r)-1)}{\sinh (\kappa r)},
$$

where, we recall, $P$ is the bulk thermodynamic pressure. (For a discussion of the different definitions of pressure in the OCP in the hyperbolic plane, see Fantoni et al.[18].) We shall show that the above equation of state reduces to known results in the appropriate limits.

Note that generalized Stillinger-Lovett sum rules are satisfied by the OCP in the hyperbolic plane[17], namely:

$$
\begin{aligned}
& \int d A h(r)=-1, \\
& \frac{4 \pi \rho \beta}{\kappa^{2}} \int d A h(r) \ln (\cosh (\kappa r / 2)=-1 .
\end{aligned}
$$

These rules express the fact that the system is a conductor (in which therefore electroneutrality and screening hold).

In the high-temperature limit, the Debye-Hückel approximation becomes asymptotically exact and provides an analytical expression for the pair correlation function $h(r)$ [19, 17]:

$$
h(r)=-\beta q^{2} Q_{\nu}(\cosh (\kappa r)),
$$

where $q$ is the charge of the particles and $Q_{\nu}$ is a Legendre function of the second kind with an index $\nu$ given by

$$
\nu=-\frac{1}{2}+\sqrt{\frac{1}{4}+2 \frac{\pi \beta \rho q^{2}}{\kappa^{2}}} .
$$

One can check that the expression in Eq. (35) satisfies the two above sum rules.

We can now combine the generalized virial equation of state that we have derived with the Debye-Hückel expression for $h(r)$. Inserting Eq. (35) in Eq. (32) leads to the following equation of state:

$$
\frac{\beta P}{\rho}=1-\frac{\pi\left(\beta q^{2}\right)^{2} \rho}{\kappa^{2}} \int_{1}^{\infty} d x \frac{Q_{\nu}(x)}{x+1} .
$$

For a fixed nonzero curvature $(\kappa>0)$, Eq. (36) shows that $\nu \rightarrow 0$ in the hightemperature limit $(\beta \rightarrow 0)$; knowing that $Q_{0}(x)=\frac{1}{2} \ln \left(\frac{1+x}{1-x}\right)$ and using that

$$
\int_{1}^{\infty} \frac{\frac{1}{2} \ln \left(\frac{1+x}{1-x}\right)}{1+x}=\frac{\pi^{2}}{12},
$$


one finally obtains that

$$
\frac{\beta P}{\rho}=1-\frac{\pi^{3}\left(\beta q^{2}\right)^{2} \rho}{12 \kappa^{2}}
$$

This expression coincides with the dominant term in the high-temperature limit of the virial expansion derived by Jancovici and Tellez[19].

On the other hand, when $\kappa \rightarrow 0$ (faster than $\beta^{1 / 2}$ ), $\sim \sqrt{2 \pi \beta \rho q^{2}} / \kappa \rightarrow+\infty$ and one has

$$
\int_{1}^{\infty} d x \frac{Q_{\nu}(x)}{1+x} \sim \frac{1}{2 \nu^{2}}
$$

The pressure is then given by

$$
\frac{\beta P}{\rho}=1-\frac{\beta q^{2}}{4}
$$

which corresponds to the result in the Euclidean limit 48].

We next consider the specific value of the temperature $T=q^{2} /\left(2 k_{B}\right)$, usually expressed in terms of the coupling parameter $\Gamma=\beta q^{2}=2$. After a mapping onto a non-Hermitian fermionic field theory, Hastings [49] has obtained the exact expression of the pair correlation function for Coulomb systems in the hyperbolic plane for this value of the coupling. For the OCP, this provides

$$
h(r)=-\frac{1}{\cosh (\kappa r / 2)^{8 \pi \rho / \kappa^{2}+2}} .
$$

After introducing the change of variable $x=\cosh (\kappa r / 2)$ and inserting Eq. (42) in Eq. (32), we obtain

$$
\frac{\beta P}{\rho}=1-\frac{2 \pi \beta q^{2} \rho}{\kappa^{2}} \int_{1}^{\infty} d x \frac{1}{x^{3+\frac{8 \pi \rho}{\kappa^{2}}}} .
$$

Since $\beta q^{2}=2$, the bulk thermodynamic pressure is finally given by

$$
\frac{\beta P}{\rho}=\frac{2 \pi \rho+\kappa^{2}}{4 \pi \rho+\kappa^{2}} .
$$

In the low-density limit, this gives $\beta P / \rho=1-\left(2 \pi / \kappa^{2}\right) \rho+O\left(\rho^{2}\right)$, which leads to the same second virial coefficient as found in Ref. [19].

When $\kappa \rightarrow 0$, one recovers from Eq. (44) the exact Euclidean limit

$$
\frac{\beta P}{\rho}=\frac{1}{2}
$$

Conversely when $\kappa \rightarrow \infty$, the pressure goes to the ideal-gas limit. This result seems to be quite general, namely: the influence of the inter-particle interactions vanishes in the large-curvature limit. 


\subsection{Two-component plasma (TCP)}

The TCP has been investigated in Euclidean space [50, 51, 52, 53, 54]. For point particles, the plasma is stable in the high-temperature conducting phase, for a coupling parameter $\beta q^{2}<2$. Adding a small hard-core repulsive potential allows one to obtain a stable phase at lower temperatures, up to a coupling $\beta q^{2}=4$ where a Kostelitz-Thouless transition between a conducting phase and a dipolar phase takes place. As for the OCP, the model is solvable for $\beta q^{2}=2$ in various geometries [55, 56, 57].

The bulk thermodynamic pressure of the TCP in the hyperbolic plane is expressable as

$$
\frac{\beta P}{\rho}=1+\frac{\pi \beta q^{2} \rho}{\kappa} \int_{0}^{\infty} d r\left(h_{++}(r)-h_{+-}(r)\right) \frac{(\cosh (\kappa r)-1)}{\sinh (\kappa r)},
$$

where $h_{++}(r)$ and $h_{--}(r)$ denote the pair correlation functions between particles of equal charges and particles of opposite charges, respectively. In the high-temperature/lowcoupling limit, the Debye-Hückel approximation provides an accurate description of the conducting phase. For the TCP, the linearized Poisson equation is similar to that of the OCP. The pair correlation functions are then given by

$$
h_{++}(r)=-h_{+-}(r)=-\frac{\beta q^{2}}{2} Q_{\nu}(\kappa r) .
$$

After inserting Eq.(47) in Eq.(46), one obtains an equation of state in the Debye-Hückel approximation which is equal to that of the OCP in the same approximation, Eq. (39).

For $\beta q^{2}=2$, Jancovici and Tellez[19] have obtained the exact expressions of the pair correlation functions of the TCP (in the limit of very small hard-core interactions). However, the calculation of the equation of state via Eq. (46) becomes much more involved than for the OCP without bringing much new insight. Therefore, we have not pursued in this direction.

\section{Short-range potentials}

\subsection{The hard-disk fluid}

As mentioned in Introduction, the hard-disk fluid has been considered in the context of geometrical frustration. Approximate equations of state combining the low-density expansion and a description of close or nearly close packings have been proposed [20, 21 , 22 .

We have solved the PY integral equation, which is known to be generally better than the HNC one for hard spherical particles [32], for a large density range. Fig. 1 shows the resulting radial distribution function $g(r)$ for different values of the curvature

at an area fraction $\phi=\rho \frac{2 \pi(\cosh (\kappa \sigma / 2)-1)}{\kappa^{2}} \simeq 0.55$ (recall that the freezing area fraction in the Euclidean plane is around $0.7[58]$ ). We have also plotted the Euclidean counterpart corresponding to $\kappa=0$. It is striking that for a range of curvature parameter, $\kappa \sigma$ between 0 and 0.5 , all curves are essentially superimposable: in this range, the pair 


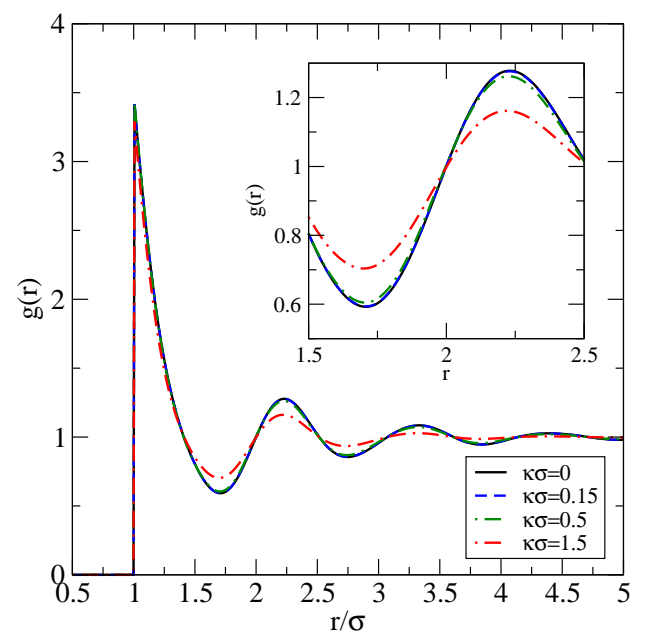

Figure 1. Radial distribution function $g(r)$ obtained from the PY equation at an area fraction $\phi=0.55$ for various values of the curvature: $\kappa \sigma=0,0.15,0.5,1.5$. Only for $\kappa=1.5$, namely a radius of curvature smaller than the particle diameter, the structure displays significant deviations from the Euclidean case. The inset zooms in the region of the first minimum and second maximum: $g(r)$ for $\kappa \sigma=0.15$ and for the Euclidean case are indistinguishable, whereas for $\kappa \sigma=0.5$, the extrema are slightly less pronounced.

distribution function $g(r)$ is rather insensitive to curvature. An evolution is on the other hand clearly seen for stronger curvatures, e.g. $\kappa \sigma=1.5$.

From the PY correlation function, we have calculated the equation of state through Eqs. (14), (24), and (25). The results are illustrated in Fig. 2, Similarly to the Euclidean case, the compressibility route gives a higher bulk thermodynamic pressure than the virial route, and the linear combination given in Eq. (25) lies in between. Comparison with the molecular dynamics data of Ref. [20, 21] and with the equation of state proposed in Ref. 22] is performed in Fig. 3. At low area fractions simulation data and predictions share the same behavior controlled by the second virial coefficient. However, the situation deteriorates for even moderate area fractions. (Note that the number of atoms in the simulation cell with periodic boundary condition is very small, always less than 10.)

It is interesting to investigate the local order of the hard-disk fluid. The geometric frustration induced by the negative curvature describes the impossibility of extending the local order present in the dense fluid to tile the whole space. This is true for a nonzero but small enough frustration for which the local order remains that of the Euclidean plane, i.e. the hexagonal order. As stressed by Rubinstein and Nelson[26], there is a series of increasing curvature parameters $\kappa \sigma$ for which heptagonal, octagonal, etc..., local order can tile space without frustration. Two points however are worth stressing. First, such regular tilings are only a few of all possible tesselations of the hyperbolic plane. 

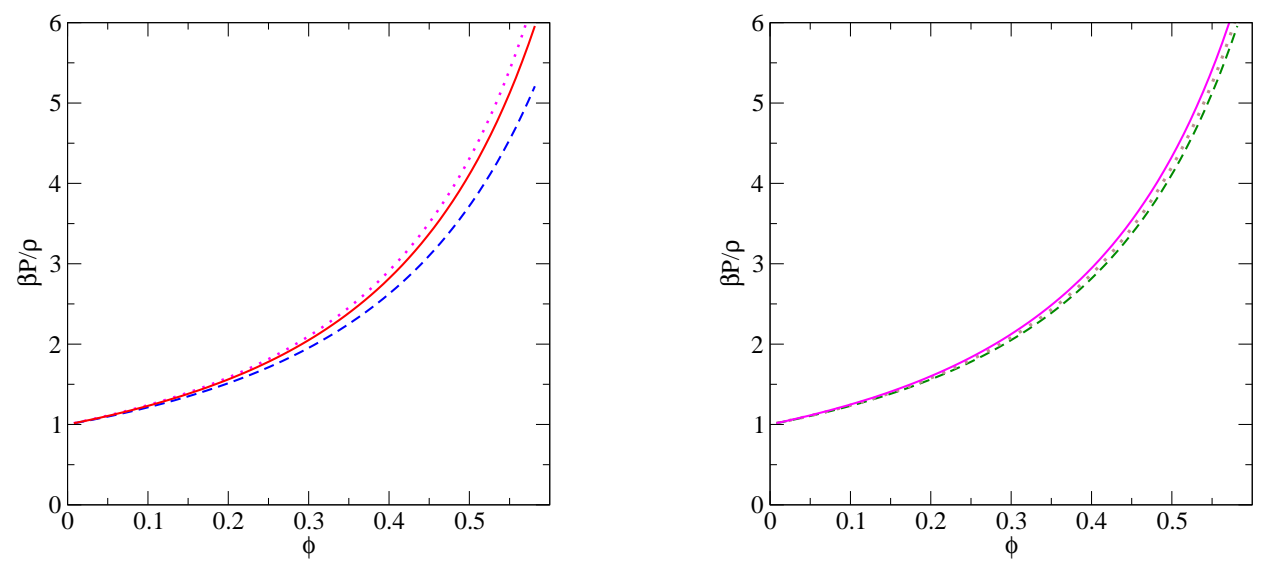

Figure 2. PY equation of state of the hard-disk fluid in $H^{2}$ versus area fraction $\phi$. (a) $\kappa \sigma=0.15$ : the upper curve corresponds to the compressibility route, the lower curve to the virial route, and the middle curve to the empirical rule in Eq. (25). (b) PY equation of state obtained from Eq. (25) for different curvatures: $\kappa \sigma=0.15,0.7,1.06$ from bottom to top.

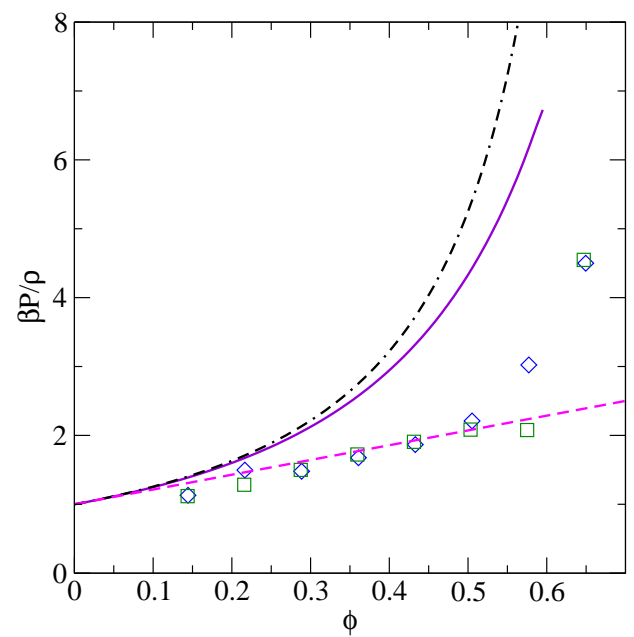

Figure 3. Equation of state of the hard-disk fluid in $H^{2}$ versus area fraction $\phi$ : comparison between the PY result combined with Eq. (25) (for $\kappa \sigma=1.06$ ) and the simulation results of Ref. 21] for $\kappa \sigma=1.060$ (green squares) and $\kappa \sigma=1.062$ (blue diamonds). The dash-dotted line is the prediction of Ref. [22] and the dashed line corresponds to the equation of state truncated at the second virial coefficient.

Modes and Kamien[20, 21] have rather focused on so-called isostatic regular packings, i.e. tilings of $H^{2}$ in which each atom has 4 neighbors (the $\{4,5\}$ tesselation for instance 
can be realized as a close packing of disks for a curvature intermediate between the two curvatures studied in their simulations: $\kappa \sigma=1.0612$ ). However, such isostatic packings have a small density compared to the heptagonal $\{7,3\}$, octagonal $\{8,3\}$, etc..., tilings considered in Ref. [26]. Second, the local order in the equilibrium hard-disk fluid has not actually been investigated as a function of curvature.

Our study sheds some light on the latter question. From the knowledge of the radial distribution function, we have calculated the average coordination number $\bar{Z}$ via the formula

$$
\bar{Z}=2 \pi \rho \int_{\sigma}^{\sigma_{m}} d r \frac{\sinh (\kappa r)}{\kappa} g(r),
$$

where $\sigma_{m}$ corresponds to the distance between particles when $g(r)$ attains the first minimum beyond the first peak. The results are plotted as a function of area fraction for different values of the curvature in Fig. 4. As expected, the average coordination number increases with density and seems to saturate (we cannot access close packing values with the approximate PY integral equation). It also increases with the curvature parameter. The values at high density are in semi-quantitative agreement with the prediction of Rubinstein and Nelson [26] based on a fictive "ideal" random close packing,

$$
\bar{Z}(\kappa \sigma)=\frac{\pi}{\arcsin \left(\frac{1}{2 \cosh \left(\frac{\kappa \sigma}{2}\right)}\right)},
$$

a prediction that interpolates between the exact values of the tesselations: $\bar{Z}=6$ for the $\{6,3\}$ tiling when $\kappa=0, \bar{Z}=7$ for the $\{7,3\}$ tiling when $\kappa \sigma=1.0905$. ,

$\bar{Z}=8$ for the $\{8,3\}$ tiling when $\kappa \sigma=1.5285 .$. , etc.... The coordination number is clearly overestimated for large curvatures, e.g. $\kappa \sigma=1.06$, in the PY approximation and we suspect that the validity of the approximate closures quite generally gets worse as curvature increases.

\subsection{The truncated Lennard-Jones potential}

The Lennard-Jones pair potential,

$$
u(r)=4 \epsilon\left[\left(\frac{\sigma}{r}\right)^{12}-\left(\frac{\sigma}{r}\right)^{6}\right]
$$

is commonly used to model liquids in Euclidean space. However, as shown in section 3, its power-law nature makes it inappropriate for hyperbolic geometry as no thermodynamic limit would be reachable. Physically, the $(\sigma / r)^{6}$ term arises from London dispersion forces and its electrostatic origin suggests that the dependence on the geodesic distance should be modified in the hyperbolic plane. Indeed, even the Coulomb potential that (logarithmically) increases with distance in two-dimensional Euclidean space is found to decay exponentially in the hyperbolic plane with an intrinsic screening length which is provided by the radius of curvature $\kappa^{-1}$. As for the $(\sigma / r)^{12}$ term, it is merely a convenient way to mimic a steeply repulsive potential between atoms and its algebraic character has no physical foundation. 


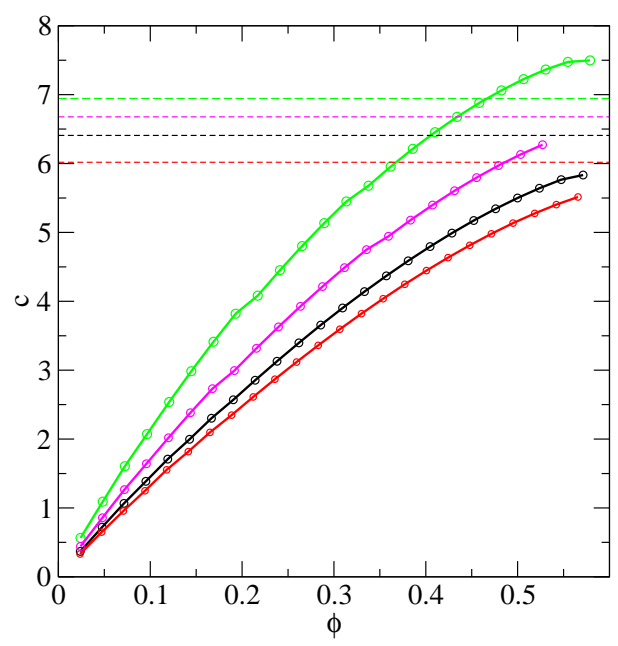

Figure 4. Average coordination number versus area fraction $\phi$ for different values of the curvature, as obtained from the PY equation. From bottom to top: $\kappa \sigma=$ $0.15,0.7,0.9,1.06$. The dashed horizontal lines indicate the maximum values predicted by Rubinstein and Nelson[26].

To preserve a proper thermodynamic limit in $H^{2}$, the simplest procedure is to truncate the Lennard-Jones potential, Eq. (50), beyond some cut-off distance $r_{c}$ : this should not significantly alter the behavior of the liquid, provided $r_{c}$ is less than the radius of curvature $\kappa^{-1}$. This cut-off procedure is anyhow most often employed in the Euclidean case in order to save computer time, and this will facilitate the comparison between the results in Euclidean and hyperbolic planes. In the Euclidean plane, Glandt[59, 60] has obtained the numerical solution of the PY and HNC integral equations for the LennardJones interaction potential. In this case, the HNC equation becomes significantly more accurate than the PY equation as the density increases. (A comparison with simulations has been performed in Ref.61].)

In the present work, we have numerically solved both the PY and HNC integral equations for a large range of temperature and density. As in the case of the hard-disk fluid, we observe a very small evolution of the structure with the curvature and the differences between the PY and HNC closures are very small too up $\kappa \sigma \simeq 0.5$ (curves not worth showing here).

We first focus on the region of density and temperature close to the gas-liquid critical point. A simple iterative procedure (Picard method) is then inefficient for converging towards the solution of the integral equation. It is necessary to proceed with a more sophiscated iterative scheme. We have combined the Newton method with a conjugate gradient procedure 62, 63] (which avoids the operator inversion otherwise present in the Newton method[64]). We have adapted this method to hyperbolic geometry. Details are given in Appendix B. 


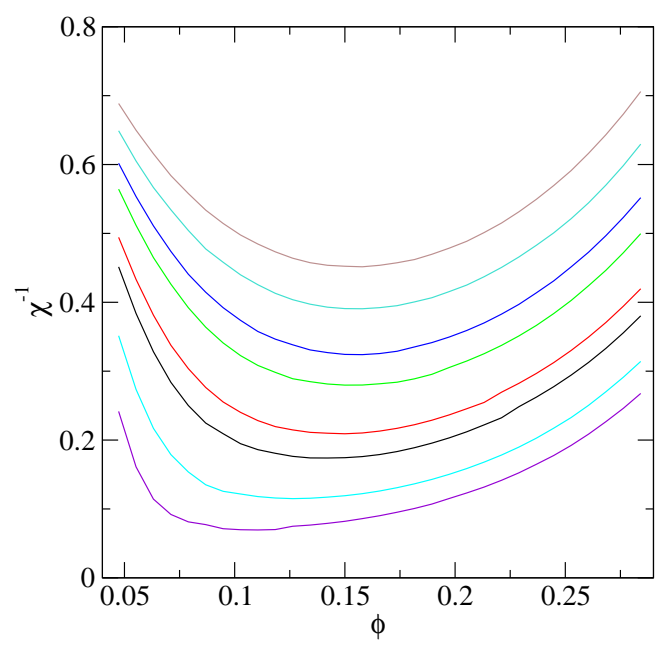

Figure 5. Inverse of the isothermal compressibility versus area fraction $\phi$ for the truncated Lennard-Jones fluid in the PY approximation near but above the critical temperature: from top to bottom , $T=0.640,0.610,0.580,0.560,0.530,0.515,0.490,0.472$. The curvarture parameter is $\kappa \sigma=0.5$.

We illustrate these results for the PY closure and a reduced curvature $\kappa \sigma=0.5$. Differents isotherms above the critical temperature are shown in Fig 5 , The inverse of the susceptibility reaches a minimum at each temperature and the successive minima approach zero as one gets closer to the critical point. Figure 6a displays the pair correlation functions at a reduced temperature $T=0.515$ for several area fractions; the log-linear plot clearly indicates that the decay is always exponential and faster than $\exp (-\kappa r)$ (dotted straight line). A more stringent test of the long-distance behavior of the pair correlation function $h(r)$ is shown in Fig. 6b , where $\exp (\kappa r) h(r)$ is plotted as a function of the distance $r$. One can see the approach to a plateau for large enough $r$ as one approaches the critical point. (The slowest decay corresponds to the area fraction at which the compressibility is maximum.)

We have also considered the liquid at a rather high density. Recently, we have performed extensive molecular dynamics simulations of the monodisperse truncated Lennard-Jones liquid in the hyperbolic plane (with periodic boundary conditions) [30]. We have shown that a small curvature indeed prevents long-range hexagonal (or hexatic) ordering and leads to glass formation as temperature is lowered. Most results were obtained for a density $\rho \sigma^{2}=0.852$. Fig. 7 displays the radial distribution function $g(r)$ at an area fraction $\phi=0.669$ and a high temperature $T=3.259$ for a curvature parameter $\kappa \sigma=0.1\left(\rho \sigma^{2}\right.$ is then equal to 0.852$)$. At this state point, the PY solution provides a slightly better description of the simulation data than the HNC equation: the HNC equation underestimates the structure of the liquid at short distances (see the inset of Fig. (7). When the temperature is lowered to $T=1.885$ (i.e., still above the freezing 

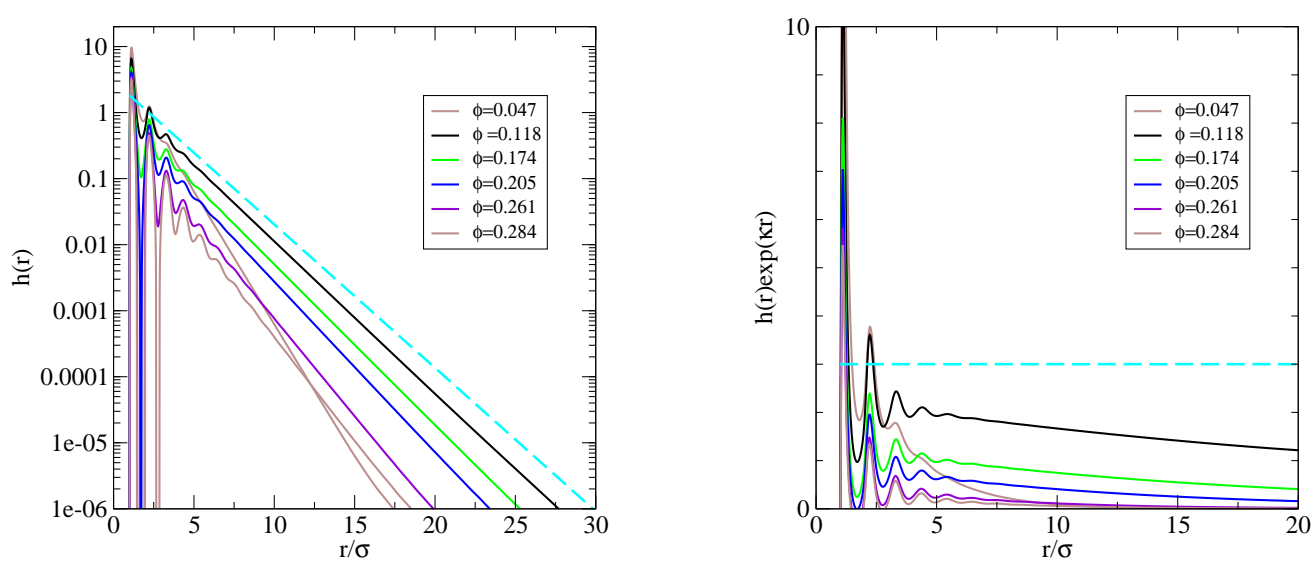

Figure 6. (a) Log-linear plot of pair correlation function $h(r)=g(r)-1$ in the PY approximation for $T=0.472$. The behavior as a function of $\phi$ is non monotonous and the slowest decay is for $\phi=0.118$, which corresponds to the maximum of the compressibility. The dotted line is $\exp (-\kappa r)$. (b) Same data multiplied by $\exp (\kappa r)$ : a convergence towards a constant plateau at large $r$ is clearly visible as one approaches the critical point. The curvature parameter is $\kappa \sigma=0.5$.

temperature in the Euclidean plane which is around $T^{*} \simeq 0.75$ at this density[30]), the HNC and PY closures are now of comparable agreement with the simulation data, but the maximum of the first peak is overestimated. Overall, the agreement appears good with either the PY or the HNC prediction.

\section{Conclusion}

We have formulated a statistical-mechanical treatment for describing the thermodynamics and the structure of fluids embedded in the hyperbolic plane, focusing on the bulk behavior. We have applied the formalism to the one- and the two-component plasma models, the hard-disk fluid and the (truncated) Lennard-Jones liquid model. The negative curvature of space provides an intrinsic length that screens the long-distance behavior of the pair correlations: this is observed both for the Coulombic interactions and for the vicinity of the gas-liquid critical point. The formalism correctly reproduces the known results in the appropriate limits and, via approximate integral equations and exact thermodynamic relations, it leads to predictions that compare well with existing simulation data.

A major motivation for studying liquids in the hyperbolic plane comes from the theoretical description of jamming phenomena and glass formation in terms of geometric frustration. Frustrated local hexagonal order in negatively curved space provides a simple model to study the glass transition. Among the many theories proposed for explaining the glass phenomenology, several attribute the slowing of relaxation with 

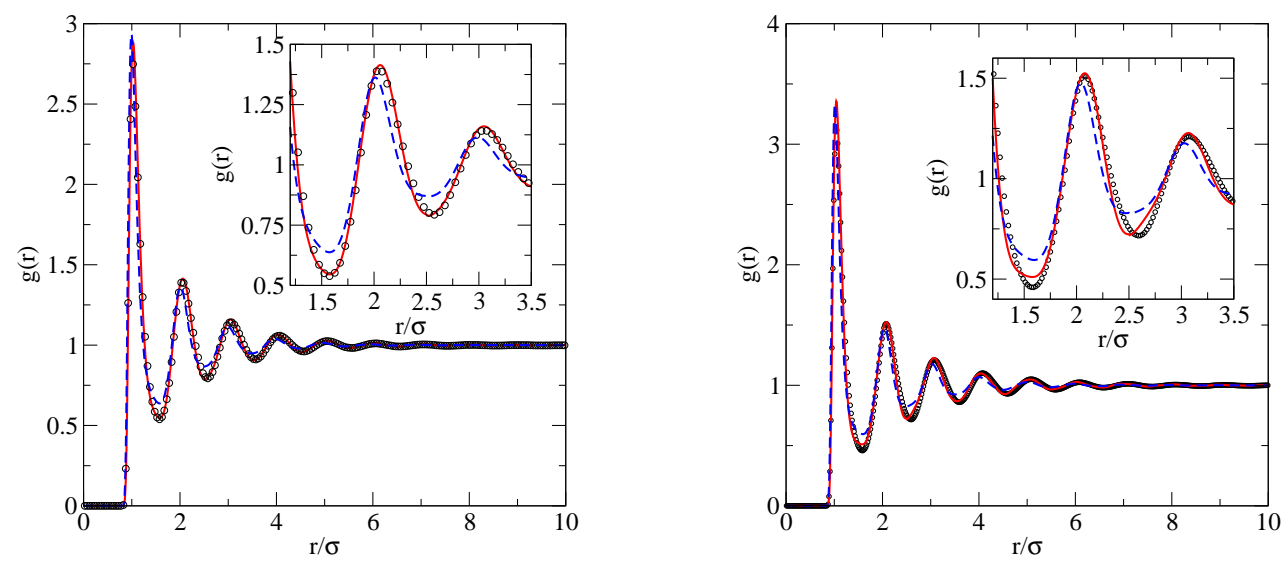

Figure 7. Radial distribution function $g(r)$ of the (truncated) Lennard-Jones liquid on $H^{2}$ for a curvature parameter $\kappa \sigma=0.1$, an area fraction $\phi=0.669$, and two different temperatures: left, $T=3.259$; right, $T=1.885$. The circles correspond to the simulation data, the full (red) curve to the PY equation, and the dashed (blue) curve to the HNC equation. The inset zooms in on the region near the first minimum and the following extrema.

decreasing temperature to the presence of a very large number, actually exponential in the system size, of metastable states of low energy or free energy [65, 66, 67]. Techniques borrowed from spin-glass theory such as the replica formalism have been applied to liquid models in three dimensions to check for the existence of such a multitude of metastable states, and evidence has been provided within mean-field-like liquid-state approximations 68, 69, 70]. Since in some sense, the hyperbolic metric induces in statistical systems some features of mean-field models, one may wonder whether a glassforming liquid in the hyperbolic plane such as the truncated Lennard-Jones model also possesses a complex free-energy landscape at low temperature. The integralequation approach developed in the present formalism can be combined with the replica method to address this point. Work in this direction is in progress.

P.V. acknowledges B. Jancovici for useful suggestions on Coulombic systems.

\section{Appendix A. Harmonic analysis in the hyperbolic plane.}

In $H^{2}$ it is possible to define a generalization of the usual spatial Fourier transform, the Fourier-Helgason transform. For a generic function $f$ of the polar coordinates $r, \phi$, it is defined as [37]

$$
\tilde{f}(a, t)=\int d r d \phi \sinh (r) e^{i a \phi} P_{-1 / 2+i t}^{a}(\cosh (r)) f(r, \phi),
$$


where $P_{-1 / 2+i t}^{a}(x)$ is a Legendre function of the first kind (conical function). The inverse Fourier-Helgason transform is given by

$$
\begin{aligned}
f(r, \phi)=\frac{1}{2 \pi} \sum_{a \in Z}(-1)^{a} \int_{t>0} d t t \tanh (\pi t) e^{-i a \phi} \\
\\
P_{-1 / 2+i t}^{-a}(\cosh (r)) \tilde{f}(a, t) .
\end{aligned}
$$

For isotropic $(S O(2)$ invariant) functions, the Fourier-Helgason transform reduces to a Mehler-Fock transform[37], the dependence on $a$ disappears and for a curvature parameter $\kappa$ one obtains

$$
\tilde{f}(k)=\frac{2 \pi}{\kappa} \int_{0}^{\infty} d r \sinh (\kappa r) P_{-1 / 2+i \frac{k}{\kappa}}(\cosh (\kappa r)) f(r) .
$$

The inverse transform is then equal to

$$
f(r)=\frac{1}{4 \pi} \int d k k \tanh \left(\pi \frac{k}{\kappa}\right) P_{-1 / 2+i \frac{k}{\kappa}}(\cosh (\kappa r)) \tilde{f}(k) .
$$

Let us show how one can recover the Euclidean limit when $\kappa \rightarrow 0$. Starting from the integral representation of the Legendre functions of the first kind[37],

$$
P_{\lambda}^{\mu}(z)=\frac{\Gamma(\mu+\lambda+1)}{2 \pi \Gamma(\lambda+1)} \int_{0}^{2 \pi} d u\left[z+\sqrt{z^{2}-1} \cos (u)\right]^{\lambda} e^{i \mu u},
$$

one obtains that

$$
\begin{aligned}
& P_{-1 / 2+i \frac{k}{\kappa}}(\cosh (\kappa r))= \\
& \frac{1}{2 \pi} \int_{0}^{2 \pi} d u[\cosh (\kappa r)+\sinh (\kappa r) \cos (u)]^{-1 / 2+i \frac{k}{\kappa}}
\end{aligned}
$$

and when $\kappa$ goes to zero, one finds that

$$
\frac{1}{2 \pi} \int_{0}^{2 \pi} d u e^{i k r \cos (u)}=J_{0}(k r)
$$

where $J_{0}(r)$ is a Bessel function of the first kind. Finally, the Fourier-Helgason transform, Eq. (A.3), becomes

$$
\tilde{f}(k)=2 \pi \int_{0}^{\infty} r d r J_{0}(k r) f(r),
$$

which corresponds to the standard Fourier transform in two dimensions.

In a similar way, the inverse Helgason transform goes to

$$
f(r)=\frac{1}{2 \pi} \int_{0}^{\infty} d k J_{0}(k r) \tilde{f}(k) .
$$

The Fourier-Helgason transform satisfies the convolution theorem: if $f$ and $g$ are $S O(2)$ invariant functions, one has

$$
\widetilde{(f * g)}(k)=\tilde{f}(k) \tilde{g}(k),
$$

where the star denotes the convolution product[71].

Finally, by using an exact Mehler-Fock transform, we illustrate the result given in Eq.(22): the Fourier-Helgason transform of a given function $f$ when $k \rightarrow 0$ is not equal 
to the integral of this function in the hyperbolic plane. We consider $f(r)=\cosh (\kappa r)^{-a}$. With the change of variable $w=\cosh (\kappa r)$, the integral of $f$ is obviously equal to

$$
\int_{1}^{\infty} d w w^{-a}=\frac{1}{1+a}
$$

whereas the Fourier-Helgason transform for $k=0$ is given by

$$
\int_{1}^{\infty} w^{a} P_{1 / 2+i t} d w=\frac{\Gamma\left(\frac{a}{2}+\frac{i t}{2}-\frac{1}{4}\right) \Gamma\left(\frac{a}{2}-\frac{i t}{2}-\frac{1}{4}\right) 2^{a-2}}{\Gamma(a) \sqrt{\pi}}
$$

where $t=k / \kappa$. The second-order expansion of Eq.(A.12) is equal to

$$
\begin{gathered}
\frac{\left(\Gamma\left(\frac{a}{2}-\frac{1}{4}\right)\right)^{2} 2^{a}}{4 \Gamma(a) \sqrt{\pi}} \\
-\frac{\left(4 \Psi\left(1, \frac{a}{2}+\frac{3}{4}\right) a^{2}-4 \Psi\left(1, \frac{a}{2}+\frac{3}{4}\right) a+\Psi\left(1, \frac{a}{2}+\frac{3}{4}\right)+16\right) 2^{a}\left(\Gamma\left(\frac{a}{2}-\frac{1}{4}\right)\right)^{2}}{16(2 a-1)^{2} \sqrt{\pi} \Gamma(a)} t^{2} \\
+O\left(t^{3}\right)
\end{gathered}
$$

Apart from the limiting case $a \rightarrow \infty$, the integral over the whole space is different from the Fourier Helgason transform at $k=0$. In addition, the function $f(r)$ has a definite integral only when $a>1$, whereas the Fourier-Helgason transform at $k=0$ is defined for $a>1 / 2$. We also note that the coefficient of the second order in the expansion in $t$ is always negative for $a>1 / 2$.

\section{Appendix B. Solving integral equations in $H^{2}$ : the numerical procedure.}

For solving integral equations, the basic method is an iterative procedure, known as the Picard method, which can be easily adapted to systems in the hyperbolic plane as follows: Starting from an initial value of $\gamma_{0}(r)=h_{0}(r)-c_{0}(r), c_{0}(r)$ is obtained by using the closure equation, Eq.(19) for PY or Eq.(20) for HNC. We next perform the Mehler-Fock transform of $c_{0}(r)$. From $\tilde{c}_{0}(k)$, and using Eq.(18), we calculate $\tilde{\gamma}_{\text {new }}(k)$ through the Ornstein-Zernike equation, namely,

$$
\tilde{\gamma}(k)=\frac{\rho \tilde{c}(k)^{2}}{1-\rho \tilde{c}(k)} .
$$

Finally, we transform back $\tilde{\gamma}_{\text {new }}(k)$ to obtain a new function $\gamma_{1}(r)$. The solution is taken as a barycenter of $\gamma_{0}(r)$ and $\gamma_{\text {new }}(r)$, namely $\gamma_{1}(r)=\alpha \gamma_{0}(r)+(1-\alpha) \gamma_{\text {new }}(r)$ where $\alpha$ is a mixing parameter. The whole procedure is repeated and we consider that convergence is reached when $\left|\gamma_{n}(r)-\gamma_{n+1}(r)\right|<\epsilon$ where $\epsilon \sim 10^{-8}$.

Note that the Mehler-Fock transform (Fourier-Helgason transform of $S O(2)$ invariant functions) is implemented by using the Legendre or conical functions $P_{-1 / 2+i t}(r)$. These functions are available in the Gnu Scientific Library and are tabulated for saving computer time. The number of points in real and reciprocal space was equal to 1600, and integration was performed with a Simpson rule. In regions of the phase diagram, far from the coexistence curves and the freezing transition line, the above procedure converges rapidly. 
However, in the vicinity of the critical point, the Picard method exhibits extremely slow convergence and a Newton method is more appropriate for solving integral equations. In addition, the equations of the Newton method are not solved by a direct inversion of the associated operator (or of the large matrix obtained after discretization of the functions), but by a more efficient procedure, the conjugate gradient methods 64, 72]: it consists in solving the equations by using an iterative method that avoids the inversion of a large matrix (or an operator). The derivation of the method is done below for the PY closure, but it can be easily modified for the HNC closure.

After differenciating Eq.(18) and (19), and letting $f(r)$ denote the Mayer function, the equations involved in the Newton method are then given by

$$
\begin{aligned}
(1-\rho \tilde{c}(k)) \delta \tilde{\gamma}(k)-\rho(\tilde{\gamma}(k)+2 \tilde{c}(k)) \delta \tilde{c}(k) & =\rho(\tilde{\gamma}(k)+\tilde{c}(k)) \tilde{c}(k)-\tilde{\gamma}(k)( \\
\delta c(r)-f(r) \delta \gamma(r) & =-c(r)+f(r)(1+\gamma(r))
\end{aligned}
$$

where $\delta c(r)(\delta \tilde{c}(k))$ and $\delta \gamma(r)(\delta \tilde{\gamma}(k))$ are solutions of the Newton equations.

By taking the Fourier-Helgason transform of Eq.(B.3), one eliminates $\delta c$ and one obtains an equation of the form

$$
A \delta \tilde{\gamma}(k)=B
$$

where $A$ is an operator such that

$$
A \tilde{g}(k)=(1-\rho \tilde{c}(k)) \tilde{g}(k)-\rho(\tilde{\gamma}(k)+2 \tilde{c}(k)) \widetilde{(f g)}(k)
$$

and $B$ is given by

$$
B=(-\tilde{c}(k)+\tilde{f}(k)+\widetilde{(f \gamma)}(k)) \rho(\tilde{\gamma}(k)+\tilde{c}(k))+\rho(\tilde{\gamma}(k)+\tilde{c}(k)) \tilde{c}(k)-\tilde{\gamma}(k) .(
$$

The adjoint operator $A^{\dagger}$ is given by

$$
A^{\dagger} \tilde{g}(k)=(1-\rho \tilde{c}(k)) \tilde{g}(k)-\rho(\widetilde{f(\gamma * g)}+2 \widetilde{f(c * g)})
$$

where $*$ denotes the convolution product.

In order to solve the linear system, Eq.(B.4 $)$, the solution is built on a sequence of functions in mutually conjugate directions. We introduce the inner product in FourierHelgason space:

$$
(\tilde{X}, \tilde{Y})=\int d k \frac{k}{\kappa} \tanh \left(\frac{\pi k}{\kappa}\right) \tilde{X}(k) \tilde{Y}(k) .
$$

Starting with the initial conditions

$$
\begin{aligned}
& R_{0}=A^{\dagger} B \\
& P_{0}=R_{0}
\end{aligned}
$$

the iterative procedure is given as follows:

- Calculate the coefficient $\alpha_{k}$,

$$
\alpha_{k}=\frac{\left(R_{k}, R_{k}\right)}{\left(A p_{k}, A p_{k}\right)} .
$$


- The residual and the solution become

$$
\begin{aligned}
& R_{k+1}=R_{k}-\alpha_{k} A^{\dagger} A P_{k} \\
& X_{k+1}=X_{k}+\alpha_{k} P_{k} .
\end{aligned}
$$

- The next conjugate direction is given by the relation

$$
P_{k+1}=R_{k+1}+\beta_{k} P_{k}
$$

with

$$
\beta_{k}=\frac{\left(R_{k+1}, R_{k+1}\right)}{\left(R_{k}, R_{k}\right)} .
$$

The iterative procedure is stopped when $\left(R_{k+1}, R_{k+1}\right)<\epsilon\left(\epsilon \sim 10^{-10}\right.$ in practice). We then take the inverse Fourier-Helgason transform of the solution $\delta \tilde{\gamma}(k)$, and with the help of Eq.(B.3), we derive $\delta c(r)$, and finally $\delta \tilde{c}(k)$ by a Fourier-Helgason transform.

We next update the functions $\gamma$ and $c$ in both real and reciprocal spaces : $\gamma_{1}=\gamma_{0}+\delta \gamma$ and $c_{1}=c_{0}+\delta c$. The inner product $b=(B, B)$ (where $B$ is given by Eq.(B.6 ) is calculated by using the updated functions $\gamma$ and $c$. So, as long as $b>\epsilon^{\prime}$ with $\epsilon^{\prime} \sim 10^{-8}$, the iterative procedure is repeated.

\section{References}

[1] R. Rietman, B. Nienhuis, and J Oitmaa. The Ising model on hyperlattices. J. Phys. A: Math. Gen., 25:6577-6592, 1992.

[2] C. Chris Wu. Ising models on hyperbolic graphs. J. Stat. Phys., 85:251-259, 1996.

[3] C. Chris Wu. Ising models on hyperbolic graphs II. J. Stat. Phys., 100:893-904, 2000.

[4] J. C. Anglès d'Auriac, R. Mélin, P. Chandra, and B. Douçot. Spin models on non-Euclidean hyperlattices: Griffiths phases without extrinsic disorder. J. Phys. A: Math. Gen., 34(4):675693, 2001.

[5] I. Benjamini and O. Schramm. Percolation in the hyperbolic plane. J. Amer. Math. Soc., 14:487$507,2001$.

[6] H. Shima and Y. Sakaniwa. The dynamic exponent of the Ising model on negatively curved surfaces. J. Stat. Mech., 2006(08):P08017, 2006.

[7] H. Shima and Y. Sakaniwa. Geometric effects on critical behaviours of the Ising model. J. Phys. A: Math. Gen., 39:4921-4933, 2006.

[8] K. Ueda, R. Krcmar, A. Gendiar, and T. Nishino. Corner transfer matrix renormalization group method applied to the Ising model on the hyperbolic plane. arXiv:0704.1949, 2007.

[9] S. K. Baek, P. Minnhagen, and B. J. Kim. Phase transition of XY model in heptagonal lattice. Europhys. Lett., 79:26002, 2007.

[10] A. Gendiar, R. Krcmar, K. Ueda, and T. Nishino. Phase transition of clock models on a hyperbolic lattice studied by corner transfer matrix renormalization group method. Phys. Rev. E, 77:041123, 2008.

[11] S. K. Baek, H. Shima, and B. J. Kim. Curvature-induced frustration in the XY model. arXiv:0811.1895.

[12] R. Krcmar, T. Iharagi, A. Gendiar, and T. Nishino. Tricritical point of the $J_{1}-J_{2}$ Ising model on a hyperbolic lattice. Phys. Rev. E, 78(6):061119, 2008.

[13] S. K. Baek, P. Minnhagen, and B. J. Kim. Percolation on hyperbolic lattices. Phys. Rev. E, 79(1):011124, 2009. 
[14] Curtis G. Callan and Frank Wilczek. Infrared behavior at negative curvature. Nuclear Physics B, 340:366-386, 1990.

[15] B. Doyon and P. Fonseca. Ising field theory on a pseudosphere. J. Stat. Mech., page P07002, 2004.

[16] L. R. A. Belo, N. M. Oliveira-Neto, W. A. Moura-Melo, A. R. Pereira, and E. Ercolessi. Heisenberg model on a space with negative curvature: Topological spin textures on the pseudosphere. Phys. Lett. A, 365:463-468, 2007.

[17] B. Jancovici and G. Téllez. Charge fluctuations for a Coulomb fluid in a disk on a pseudosphere. J. Stat. Phys., 116:205-230, 2004.

[18] R. Fantoni, B. Jancovici, and G. Téllez. Pressures for a one-component plasma on a pseudosphere. J. Stat. Phys., 112(1):27-57, 2003.

[19] B. Jancovici and G. Téllez. Two-dimensional Coulomb systems on a surface of constant negative curvature. J. Stat. Phys., 91:953-977, 1998.

[20] C. D. Modes and R. D. Kamien. Hard disks on the hyperbolic plane. Phys. Rev. Lett., 99(23):235701, 2007.

[21] C. D. Modes and R. D. Kamien. Geometrical frustration in two dimensions: Idealizations and realizations of a hard-disk fluid in negative curvature. Phys. Rev. E, 77(4):041125, 2008.

[22] M. López de Haro, A. Santos, and S. B. Yuste. Simple equation of state for hard disks on the hyperbolic plane. J. Chem. Phys., 129(11):116101, 2008.

[23] J.-F. Sadoc and R. Mosseri. Geometrical frustration. Cambridge University Press, Cambridge, 1999.

[24] D. R. Nelson. Defects and geometry in condensed matter physics. Cambridge University Press, Cambridge, 2002.

[25] D. R. Nelson. Liquids and glasses in spaces of incommensurate curvature. Phys. Rev. Lett., 50(13):982-985, 1983.

[26] M. Rubinstein and D. R. Nelson. Dense-packed arrays on surfaces of constant negative curvature. Phys. Rev. B, 28(11):6377-6386, 1983.

[27] C. Brito and M. Wyart. On the rigidity of a hard-sphere glass near random close packing. Europhys. Lett., 76(1):149-155, 2006.

[28] D. Kivelson, S.A. Kivelson, X. Zhao, Z. Nussinov, and G. Tarjus. A thermodynamic theory of supercooled liquids. Physica A, 219:27-38, 1995.

[29] G. Tarjus, S. A. Kivelson, Z. Nussinov, and P. Viot. The frustration-based approach of supercooled liquids and the glass transition: a review and critical assessment. J. Phys.: Condens. Matter, 17(50):R1143-R1182, 2005.

[30] F. Sausset, G. Tarjus, and P. Viot. Tuning the fragility of a glass-forming liquid by curving space. Phys. Rev. Lett., 101(15):155701, 2008.

[31] F. Sausset and G. Tarjus. Periodic boundary conditions on the pseudosphere. J. Phys. A: Math. Gen., 40:12873-12899, 2007.

[32] J.-P. Hansen and I. R. McDonald. Theory of simple liquids. Academic Press, 1986.

[33] J. S. Rowlinson and B. Widom. Molecular Theory of Capillarity. Clarendon, Oxford, 1982.

[34] E. Kierlik, M. L. Rosinberg, G. Tarjus, and P. Monson. The pressure of a fluid confined in a disordered porous material. J. Chem. Phys., 103(10):4256-4260, 1995.

[35] G. Stell. Correlation functions and their generating functionals,. In Phase Transitions and Critical Phenomena, volume 5b. C. Domb and M. S. Green (Academic, London), 1976.

[36] A. F. Beardon. The geometry of discrete groups. Springer-Verlag, New York, Heidelberg, Berlin, 1983.

[37] A. Terras. Harmonic analysis on symmeric spaces and applications. Springer-Verlag, New York, 1985.

[38] E. Leutheusser. Exact solution of the Percus-Yevick equation for a hard-core fluid in odd dimensions. Physica A, 127(3):667-676, 1984.

[39] M. Robles, M. López de Haro, and A. Santos. Percus-Yevick theory for the structural properties 
of the seven-dimensional hard-sphere fluid. J. Chem. Phys., 126(1):016101, 2007.

[40] René D. Rohrmann and Andrés Santos. Structure of hard-hypersphere fluids in odd dimensions. Phys. Rev. E, 76(5):051202, 2007.

[41] M. Robles, M. López de Haro, and A. Santos. Erratum: "equation of state of a seven-dimensional hard-sphere fluid. Percus-Yevick theory and molecular-dynamics simulations". J. Chem. Phys., 125(21):219903, 2006.

[42] M. Bishop, N. Clisby, and P. A. Whitlock. The equation of state of hard hyperspheres in nine dimensions for low to moderate densities. J. Chem. Phys., 128(3):034506, 2008.

[43] M. Adda-Bedia, E. Katzav, and D. Vella. Solution of the Percus-Yevick equation for hard disks. J. Chem. Phys., 128(18):184508, 2008.

[44] M. Adda-Bedia, E. Katzav, and D. Vella. Solution of the percus-yevick equation for hard hyperspheres in even dimensions. J. Chem. Phys., 129(14):144506, 2008.

[45] S.V. Lishchuk. Equation of state of the hard-disk fluid on a sphere from percus-yevick equation. Physica A, 369(2):266 - 274, 2006.

[46] J. M. Caillol and D. Levesque. Low-density phase diagram of the two-dimensional Coulomb gas. Phys. Rev. B, 33(1):499-509, 1986.

[47] J. Lidmar and M. Wallin. Monte Carlo simulation of a two-dimensional continuum Coulomb gas. Phys. Rev. B, 55(1):522-530, 1997.

[48] A. Torres and G. Téllez. Finite-size corrections for Coulomb systems in the Debye-Hückel regime. J. Phys. A: Math. Gen., 37(6):2121-2137, 2004.

[49] M.B. Hastings. Non-hermitian fermion mapping for one-component plasma. J. Stat. Phys., 90(1):311-326, 1998.

[50] J.P. Hansen and P. Viot. Pair correlations and internal energy of the two-dimensional Coulomb gas. Phys. Lett. A, 95(3-4):155-158, 1983.

[51] L. Samaj and I. Travenec. Thermodynamic properties of the two-dimensional two-component plasma. Journal of Statistical Physics, 101(3):713-730, November 2000.

[52] L. Samaj. Universal finite-size effects in the two-dimensional asymmetric coulomb gas on a sphere. Physica A: Statistical Mechanics and its Applications, 297(1-2):142 - 156, 2001.

[53] L. Samaj and B. Jancovici. Large-distance behavior of particle correlations in the two-dimensional two-component plasma. J. Stat. Phys., 106:301-321, 2002.

[54] L. Samaj and B. Jancovici. Density correlations in the two-dimensional coulomb gas. J. Stat. Phys., 106:323-355, 2002.

[55] M. Gaudin. Critical isotherm of a lattice plasma. J. Phys. France, 46(7):1027-1042, 1985.

[56] F. Cornu and B. Jancovici. The electrical double layer: A solvable model. J. Chem. Phys., 90(4):2444-2452, 1989.

[57] G. Téllez. Two-component plasma in a gravitational field. J. Chem. Phys., 106(20):8572-8578, 1997.

[58] A. Santos, M. López de Haro, and S. Bravo Yuste. An accurate and simple equation of state for hard disks. J. Chem. Phys., 103(11):4622-4625, 1995.

[59] E. D. Glandt and D. D. Fitts. Percus-Yevick equation of state for the two-dimensional LennardJones fluid. J. Chem. Phys., 66(10):4503-4508, 1977.

[60] E. D. Glandt and D. D. Fitts. Hypernetted-chain equation of state for the two-dimensional Lennard-Jones fluid. J. Chem. Phys., 68(10):4546-4550, 1978.

[61] R. R. Singh, K. S. Pitzer, J. J. de Pablo, and J. M. Prausnitz. Monte Carlo simulation of phase equilibria for the two-dimensional Lennard-Jones fluid in the Gibbs ensemble. J. Chem. Phys., 92(9):5463-5466, 1990.

[62] G. Zerah. An efficient Newton's method for the numerical solution of fluid integral equations. Journal of Computational Physics, 61(2):280-285, 1985.

[63] L. Belloni. Inability of the hypernetted chain integral equation to exhibit a spinodal line. The Journal of Chemical Physics, 98(10):8080-8095, 1993.

[64] M. J. Gillan. A new method of solving the liquid structure integral equations. Mol. Phys., 
38(6):1781-1794, 1979.

[65] M. Goldstein. Viscous liquids and the glass transition: A potential energy barrier picture. $J$. Chem. Phys., 51(9):3728, 1969.

[66] F. H. Stillinger. A topographic view of supercooled liquids and glass formation. Science, 267:1935, 1995.

[67] T. R. Kirkpatrick, D. Thirumalai, and Peter G. Wolynes. Scaling concepts for the dynamics of viscous liquids near an ideal glassy state. Phys. Rev. A, 40(2):1045, 1989.

[68] Y. Singh, J. P. Stoessel, and P. G. Wolynes. Hard-sphere glass and the density-functional theory of aperiodic crystals. Phys. Rev. Lett., 54(10):1059-1062, 1985.

[69] M. Mézard and G. Parisi. Thermodynamics of glasses: A first principles computation. Phys. Rev. Lett., 82(4):747, 1999.

[70] G. Parisi and F. Zamponi. Mean field theory of the glass transition and jamming of hard spheres. arXiv:0802.2180v2.

[71] B. J. Gonzalez and E. R. Negrin. Mehler-Fock transforms of generalized functions via the method of adjoints. Proc. Amer. Math. Soc., 125:3243-3253, 1997.

[72] J. Brader. Solution of the Ornstein-Zernike equation in the critical region. Int.. J. Thermophys., 27(2):394-412, 2006. 